\title{
Sleep: an emerging therapeutic target in diabetes care
}

\author{
Nishant Raizada ${ }^{1}$. S. V. Madhu ${ }^{1}$ \\ Published online: 13 February 2021 \\ (C) Research Society for Study of Diabetes in India 2021
}

The management of chronic disorders such as diabetes mellitus is a challenge for modern medicine. Although several pharmacological agents have been developed for the treatment of diabetes mellitus, most oral drugs have limited efficacy in terms of reduction in glycosylated hemoglobin (HbAlc) levels along with a gradual decline in response as beta cell failure worsens. Insulin, although effective at all ranges of hyperglycemia, is cumbersome to administer and titrate, thereby making it unsuitable for many patients with type 2 diabetes mellitus. Hence, the need for novel therapeutic modalities in diabetes cannot be overemphasized.

Type 2 diabetes mellitus has been described as a lifestyle disorder. On a background of genetic predisposition, poor lifestyle choices in terms of diet and physical activity have been implicated in the pathogenesis of type 2 diabetes mellitus. Accordingly, diabetic diet and physical activity have proven to be the cornerstone in the management of diabetes. The decline in $\mathrm{HbAlc}$ with diabetic diet and physical activity exceeds that seen with individual pharmacological agents with the exception of insulin. However, diet and physical activity are not the only lifestyle factors affecting diabetes. Among the other lifestyle factors, sleep has unfortunately received less attention until recently. While obstructive sleep apnea has been well recognized as a risk factor for several metabolic diseases, the role of sleep disorders other than obstructive sleep apnea seems to be equally important.

Sleep disorders are commonly seen in diabetes. The prevalence of sleep disturbances in clinic-based populations of type 2 diabetes mellitus patients ranges from 33 to $73 \%$ [1-4]. Data suggests that sleep and sleep disorders influence diabetes at multiple points in the natural history of the disease. Sleep duration of less than $8 \mathrm{~h}$ has been shown to increase the risk of developing type 2 diabetes mellitus in a Chinese cohort

S. V. Madhu

drsvmadhu@gmail.com

1 Department of Endocrinology, Center for Diabetes, Endocrinology and Metabolism, University College of Medical Sciences \& GTB Hospital, New Delhi, India and a risk score incorporating sleep duration has also been developed [5]. Sleep efficiency (time spent as sleep as a percentage of time spent in bed) of less than $80 \%$ was associated with type 2 diabetes mellitus with an odds ratio close to two in the Sleep Heart Heath Study [6]. A survey in Taiwanese public servants also revealed an association between shorter sleep duration and type 2 diabetes [7].

Gestational diabetes mellitus (GDM) which is a common precursor of type 2 diabetes mellitus in women appears to have links with sleep disturbances. In this issue, Wang et al. report that sleep duration of $10 \mathrm{~h}$ or more along with prepregnancy obesity is a strong risk factor for GDM. Both too much and too little night sleep and too much day time napping is associated with GDM in Chinese women [8]. The risk of GDM increased by $32 \%$ in those with shorter sleep duration in another study from China [9]. A systematic review and meta-analysis also found that poor sleep quality increases risk of GDM [10].

Sleep disorders appear to be associated with poor glycemic control in patients already suffering from diabetes mellitus. Sleep fragmentation was associated with worse HbA1c and fasting plasma glucose in a black population in the USA [11]. Poor sleep quality was also associated with higher $\mathrm{HbAlc}$, BP, and obesity [12]. Even in patients who did not report any subjective sleep disturbances, a negative correlation between objective parameters of sleep quality and $\mathrm{HbA} 1 \mathrm{c}$ was found [6].

Furthermore, sleep disorders have not only been associated with complications of diabetes but also with mortality risk. In a large cohort of adults from the USA, risk of mortality in type 2 diabetes mellitus patients (compared to non-diabetics) increased from 1.84 with a sleep duration of $7 \mathrm{~h}$ to 2.78 and 3.67 for 5 and $10 \mathrm{~h}$ respectively [13]. Similarly, data from the Korean National health insurance program found that newly diagnosed type 2 diabetes mellitus patients with sleep disturbances have a higher incidence of cardiovascular disease and all-cause mortality [14]. Poor sleep quality has also been shown to be associated with microvascular complications such as diabetic retinopathy [15]. 
Although data in type 1 diabetes mellitus is limited, the role of sleep disorders cannot be discounted. Reduced sleep duration and quality were associated with higher glycemic variability along with greater stress and depression in type 1 diabetes mellitus [16]. In another study, type 1 diabetes mellitus patients with poor sleep quality had higher total and LDL cholesterol as compared to good sleepers [17].

While the high prevalence of sleep disorders and their impact on diabetes risk, glycemic control, diabetes complications, and mortality paint a gloomy picture, the proverbial cloud does have a silver lining. In this issue, Ghadimi et al. have reported that administration of ellagic acid, a polyphenol known to be present in fruits and nuts, leads to a significant improvement in sleep quality in patients with type 2 diabetes mellitus. Although the mechanism of this improvement is unclear and a subject for further research, the authors hypothesize that the antioxidant properties of ellagic acid may contribute to reduction in reactive oxygen species and chemicals such as malondialdehyde - these molecules may play a role in sleep disturbances. The results of this double blind randomized trial are encouraging despite being a short duration study carried out in a small number of patients and could mark the beginning of a new approach in the management of diabetic patients if these can be replicated in larger studies with longer follow-up.

Patients with sleep disturbances due to coexisting obstructive sleep apnea (OSA) benefit from continuous positive airway pressure (CPAP) ventilation. CPAP in patients with type 2 diabetes and OSA have shown to improve HbA1c and reduce insulin resistance [18]. Even in patients with wellcontrolled diabetes and undiagnosed OSA, CPAP improves daytime sleepiness. The Diabetes Sleep Treatment Trial, which is currently ongoing, will compare CPAP with shamCPAP with respect to improvement in glycemic control in patients with type 2 diabetes [19]. Liraglutide has shown to reduce apnea hypopnea index in patients with diabetes and OSA, presumably by inducing weight loss [20].

In patients who do not have OSA but still have sleep disturbances, several non-pharmacological interventions have been documented to be beneficial. In a randomized control trial, improvement in sleep quality in type 2 diabetes mellitus patients using cognitive behavioral therapy led to improvement in $\mathrm{HbA} 1 \mathrm{c}$ to the tune of $0.9 \%$ [21]. A structured sleep education program in patients with type 2 diabetes who were sleeping after midnight leads to significant improvements in their sleep quality and glycemic control [22]. Nutritional ketosis under a continuous care intervention has also shown to improve sleep quality in type 2 diabetes [23].

Aerobic exercise training in type 2 diabetes has shown to improve sleep quality as well as quality of life [24]. However ,both aerobic and resistance exercise can predispose to hypoglycemia and cause sleep loss in type 1 diabetes [25]. Management of hypoglycemias by utilizing flash glucose monitoring can improve sleep disturbances [26]. Closed loop continuous subcutaneous insulin infusion (CSII) systems have also been studied regarding impact on sleep quality in type 1 diabetes although data suggests that they may not offer any advantage over sensor augmented pumps in this regard [27].

Treatment of both painless and painful neuropathy along with restless legs syndrome can also improve sleep disturbances [28]. In patients with diabetic neuropathic pain, mirogabalin has shown reduction in average daily sleep interference scores [29]. Sustained release oxycodone has also led to improvement in sleep quality in patients with type 2 diabetes and severe painful neuropathy [30]. Other drugs like mexiletine and gabapentin may also improve sleep disturbances by ameliorating pain in diabetic neuropathy [31].

Vitamin D supplements [32] and reservetrol [33] have been tried in healthy adults with sleep disorders with variable success although these have not been specifically evaluated in diabetic subjects.

Sleep and interventions to control sleep disorders have the potential to become an important component of diabetes care in future. Considering the limitations of current diabetes management, these novel strategies may well be the much needed ray of hope.

\section{References}

1. Sridhar GR, Madhu K. Prevalence of sleep disturbances in diabetes mellitus. Diabetes Res Clin Pract. 1994;23(3):183-6.

2. Skomro RP, Ludwig S, Salamon E, Kryger MH. Sleep complaints and restless legs syndrome in adult type 2 diabetics. Sleep Med. 2001;2(5):417-22.

3. Khorasani ZM, Ravan VR, Hejazi S. Evaluation of the prevalence of sleep disorder among patients with type 2 diabetes mellitus referring to Ghaem hospital from 2016 to 2017. Curr Diabetes Rev. 2020.

4. Khalil M, Power N, Graham E, Deschênes SS, Schmitz N. The association between sleep and diabetes outcomes - a systematic review. Diabetes Res Clin Pract. 2020;161:108035.

5. Liu X, Li Z, Zhang J, Chen S, Tao L, Luo Y, et al. Novel Risk Score for Type 2 Diabetes Containing Sleep Duration: A 7-Year Prospective Cohort Study among Chinese Participants. J Diabetes Res. 2020. https://doi.org/10.1155/2020/2969105.

6. Yan B, Zhao B, Fan Y, Yang J, Zhu F, Chen Y, et al. The association between sleep efficiency and diabetes mellitus in communitydwelling individuals with or without sleep-disordered breathing. J Diabetes. 2020;12(3):215-23.

7. Shih DP, Lin PY, Liang WM, Tseng PC, Kuo HW, Wang JY. Sleep Duration and Effort-Reward Imbalance (ERI) Associated with Obesity and Type II Diabetes Mellitus (T2DM) among Taiwanese Middle-Aged Public Servants. Int J Environ Res Public Health. 2020. https://doi.org/10.3390/ijerph17186577.

8. Wang S, Xu L, Jonas JB, You QS, Wang YX, Yang H. Prevalence and associated factors of dyslipidemia in the adult Chinese population. PLOS ONE. 2011;6(3):e17326.

9. Du M, Liu J, Na H, Zhao Z, Luo S, Wang H. Association between sleep duration in early pregnancy and risk of gestational diabetes mellitus: A prospective cohort study. Diabetes Metab. 2020. https:// doi.org/10.1016/j.diabet.2020.101217. 
10. Zhu B, Shi C, Park CG, Reutrakul S. Sleep quality and gestational diabetes in pregnant women: a systematic review and meta-analysis. Sleep Med. 2020;67:47-55.

11. Yano Y, Gao Y, Johnson DA, Carnethon M, Correa A, Mittleman MA, et al. Sleep Characteristics and Measures of Glucose Metabolism in Blacks: The Jackson Heart Study. J Am Heart Assoc. 2020. https://doi.org/10.1161/JAHA.119.013209.

12. Bener A, Al-Hamaq AOAA, Agan AF, Öztürk M, Ömer A. Sleeping disturbances and predictor risk factors among type 2 diabetic mellitus patients. Ann Afr Med. 2020;19:230-6.

13. Wang Y, Huang W, O'Neil A, Lan Y, Aune D, Wang W, et al. Association between sleep duration and mortality risk among adults with type 2 diabetes: a prospective cohort study. Diabetologia. 2020;63:2292-304.

14. Choi Y, Choi JW. Association of sleep disturbance with risk of cardiovascular disease and all-cause mortality in patients with new-onset type 2 diabetes: data from the Korean NHIS-HEALS. Cardiovasc Diabetol. 2020;19(1):61.

15. Dutta S, Ghosh S, Ghosh S. Association of sleep disturbance with diabetic retinopathy. Eur J Ophthalmol. 2020;1120672120974296.

16. Rechenberg K, Griggs S, Jeon S, Redeker N, Yaggi HK, Grey M. Sleep and glycemia in youth with type 1 diabetes. J Pediatr Health Care Off Publ Natl Assoc Pediatr Nurse Assoc Pract. 2020;34(4): 315-24.

17. de Mattos ACMT, Campos YS, Fiorini VO, Sab Y, Tavares BL, Velarde LGC, et al. Relationship between sleep disturbances, lipid profile and insulin sensitivity in type 1 diabetic patients: a crosssectional study. Arch Endocrinol Metab. 2020;64(4):412-7.

18. Martínez-Cerón E, Barquiel B, Bezos AM, Casitas R, Galera R, García-Benito C, et al. Effect of Continuous Positive Airway Pressure on Glycemic Control in Patients with Obstructive Sleep Apnea and Type 2 Diabetes. A Randomized Clinical Trial. Am J Respir Crit Care Med. 2016;194:476-85.

19. Chasens ER, Atwood CW, Burke LE, Korytkowski M, Stansbury $\mathrm{R}$, Strollo PJ, et al. Diabetes sleep treatment trial: Premise, design, and methodology. Contemp Clin Trials. 2019;76:104-11.

20. Blackman A, Foster GD, Zammit G, Rosenberg R, Aronne L, Wadden $\mathrm{T}$, et al. Effect of liraglutide $3.0 \mathrm{mg}$ in individuals with obesity and moderate or severe obstructive sleep apnea: the SCALE Sleep Apnea randomized clinical trial. Int J Obes 2005. 2016;40(8): 1310-9.

21. Zuo X, Dong Z, Zhang P, Zhang P, Chang G, Xiang Q, et al. Effects of cognitive behavioral therapy on sleep disturbances and quality of life among adults with type 2 diabetes mellitus: A randomized controlled trial. Nutr Metab Cardiovasc Dis. 2020;30:1980-8.

22. Li M, Li D, Tang Y, Meng L, Mao C, Sun L, et al. Effect of diabetes sleep education for T2DM who sleep after midnight: a pilot study from China. Metab Syndr Relat Disord. 2018;16(1):13-9.

23. Siegmann MJ, Athinarayanan SJ, Hallberg SJ, McKenzie AL, Bhanpuri $\mathrm{NH}$, Campbell WW, et al. Improvement in patientreported sleep in type 2 diabetes and prediabetes participants receiving a continuous care intervention with nutritional ketosis. Sleep Med. 2019;55:92-9.

24. S Delevatti R, Schuch FB, Kanitz AC, Alberton CL, Marson EC, Lisboa SC, et al. Quality of life and sleep quality are similarly improved after aquatic or dry-land aerobic training in patients with type 2 diabetes: a randomized clinical trial. J Sci Med Sport. 2018;21(5):483-8.

25. Reddy R, El Youssef J, Winters-Stone K, Branigan D, Leitschuh J, Castle J, et al. The effect of exercise on sleep in adults with type 1 diabetes. Diabetes Obes Metab. 2018;20:443-7.

26. Al Hayek AA, Al Dawish MA. Assessing Diabetes Distress and Sleep Quality in Young Adults with Type 1 Diabetes Using FreeStyle Libre: A Prospective Cohort Study. Diabetes Ther. 2020;11:1551-62.

27. Sharifi A, De Bock MI, Jayawardene D, Loh MM, Horsburgh JC, Berthold CL, et al. Glycemia, Treatment Satisfaction, Cognition, and Sleep Quality in Adults and Adolescents with Type 1 Diabetes When Using a Closed-Loop System Overnight Versus SensorAugmented Pump with Low-Glucose Suspend Function: A Randomized Crossover Study. Diabetes Technol Ther. 2016;18: $772-83$.

28. Choi D, Kim BY, Jung CH, Kim CH, Mok JO. Association between sleep quality and painless diabetic peripheral neuropathy assessed by current perception threshold in type 2 diabetes mellitus. Diabetes Metab J. 2020.

29. Merante D, Rosenstock J, Sharma U, Feins K, Hsu C, Vinik A, et al. Efficacy of mirogabalin (DS-5565) on patient-reported pain and sleep interference in patients with diabetic neuropathic pain: secondary outcomes of a phase II proof-of-concept study. Pain Med Malden Mass. 2017;18(11):2198-207.

30. Yao P, Meng L-X, Ma J-M, Ding Y-Y, Wang Z-B, Zhao G-L, et al. Sustained-release oxycodone tablets for moderate to severe painful diabetic peripheral neuropathy: a multicenter, open-labeled, postmarketing clinical observation. Pain Med Malden Mass. 2012;13(1):107-14.

31. Oskarsson P, Ljunggren JG, Lins PE. Efficacy and safety of mexiletine in the treatment of painful diabetic neuropathy. The Mexiletine Study Group. Diabetes Care. 1997;20(10):1594-7.

32. Majid MS, Ahmad HS, Bizhan H, Hosein HZM, Mohammad A. The effect of vitamin D supplement on the score and quality of sleep in 20-50 year-old people with sleep disorders compared with control group. Nutr Neurosci. 2018;21(7):511-9.

33. Wightman EL, Haskell-Ramsay CF, Reay JL, Williamson G, Dew $\mathrm{T}$, Zhang W, et al. The effects of chronic trans-resveratrol supplementation on aspects of cognitive function, mood, sleep, health and cerebral blood flow in healthy, young humans. Br J Nutr. 2015;114(9):1427-37.

Publisher's note Springer Nature remains neutral with regard to jurisdictional claims in published maps and institutional affiliations. 